\title{
Cannabis use and brain structural alterations of cingulate cortex in early psychosis
}

Charlotte Rapp ${ }^{\mathrm{a} 1}$, Anna Walter ${ }^{\mathrm{c}, \mathrm{d} 1}$, Erich Studerus ${ }^{\mathrm{a}}$, Hilal Bugra ${ }^{\mathrm{a}}$, Corinne Tamagni ${ }^{\mathrm{a}}$,

Michel Röthlisberger ${ }^{\mathrm{b}, \mathrm{d}}$, Stefan Borgwardt ${ }^{\mathrm{c}, \mathrm{d}}$, Jacqueline Aston ${ }^{\mathrm{a}}$, Anita Riecher-

Rössler $^{\text {a* }}$

${ }^{1}$ shared first authorship

aUniversity of Basel Psychiatric Clinics, Center for Gender Research and Early Detection, c/o University Hospital Basel, Petersgraben 4, $\mathrm{CH}-4031$ Basel, Switzerland

bUniversity Hospital Basel, Clinic for Neurosurgery, Spitalstrasse 21, CH-4031 Basel,

Switzerland

'University of Basel Psychiatric Clinics, Diagnostic and Crisis Intervention Center, c/o

University Hospital Basel, Petersgraben 4, CH-4031 Basel, Switzerland

${ }^{d}$ Medical Image Analysis Centre, University of Basel, Schanzenstrasse 55, CH-4031 Basel,

Switzerland

\section{${ }^{*}$ Corresponding author:}

Prof. A. Riecher-Rössler

University of Basel Psychiatric Clinics

Center for Gender Research and Early Detection

c/o University Hospital Basel

Petersgraben 4

$\mathrm{CH}-4031$ Basel

Switzerland

Phone: +41612655114

Fax: +41612654599

E-mail: Anita.Riecher@upkbs.ch

Keywords: First episode psychosis (FEP), At risk mental state (ARMS), Magnetic resonance imaging (MRI), Cingulum 


\section{Abstract}

As cannabis use is more frequent in psychosis patients than the general population and is known to be a risk factor for psychosis, the question arises whether cannabis contributes to recently detected brain volume reductions in schizophrenic psychoses. This study is the first to investigate how cannabis use is related to cingulum volume, a brain region involved in the pathogenesis of schizophrenia, in a sample of both at risk mental state (ARMS) and first episode psychosis (FEP) subjects. A cross-sectional MRI study of manually traced cingulum in 23 FEP and 37 ARMS subjects was performed. Cannabis use was assessed with the Basel Interview for Psychosis. By using repeated measures ANCOVAs, we investigated whether current cannabis use is associated with cingulum volume, correcting for age, gender, alcohol consumption, whole brain volume and antipsychotic medication. There was a significant three way interaction between region (anterior/posterior cingulum), hemisphere (left/right cingulum) and cannabis use (yes/no) $\left(F(1,51)=5.62\right.$, Partial $\left.\eta^{2}=0.10, p=0.022\right)$. Post hoc analyses revealed that this was due to a significant negative effect of cannabis use $\left(F(1,51)=8.96\right.$, Partial $\left.\eta^{2}=0.15, p=0.004\right)$ on volume of the posterior cingulum which was independent of the hemisphere and diagnostic group and all other covariates we controlled for. In the anterior cingulum, we found a significant negative effect only on left hemisphere $\left(F(1,51)=7.02\right.$, Partial $\left.\eta^{2}=0.12, p=0.011\right)$, which was again independent of diagnostic group.

Overall, we found negative associations of current cannabis use with grey matter volume of cingulate cortex, a region rich in CB1 receptors. As this finding has not been consistently found in healthy controls, this might suggest that both ARMS and FEP subjects might be particularly sensitive to exogenous activation of these receptors. 


\section{Introduction}

The cingulum is a functionally heterogeneous region, involved in a range of behavioural domains (Vogt et al., 1992). It represents a key structure within the limbic system and is composed of several areas which differ in respect to structure and function: the different sub divisions play an important role in emotional, cognitive, attentional, nociceptive, and motoric processing (Bush et al., 2000; Vogt et al., 1992). The anterior cingulate cortex (ACC) represents a central component of those subdivisions that control affective and cognitive functions. The central task of this brain region is the modulation of inner emotional reactions. The ACC has anatomical connections to the dorsolateral prefrontal cortex, motoric areas and the thalamus, depending on the specialised subdivision. It is an essential component of social cognition and visualizing and is mainly activated by emotional stimuli (Bush et al., 2000; Kopelman et al., 2005). The posterior cingulate cortex (PCC) is activated both through emotional and non-emotional stimuli and plays an important role in memory access, visual span and spatial orientation (Vogt et al., 1992; Vogt and Laureys, 2005; Vogt et al., 2006).

Studies of individuals with genetic or clinical risk for psychosis have indicated that the cingulum might be involved in the pathogenesis of schizophrenia (for review, see Borgwardt et al., 2009; Fusar-Poli et al., 2008; Fusar-Poli et al., 2011; Smieskova et al., 2010). A recent study from our group reported that At Risk Mental State (ARMS) (Yung et al., 2005) subjects had significantly reduced left caudal ACC volume compared to healthy controls and within ARMS, those who later made the transition to psychosis (ARMS-T), showed significantly reduced whole right cingulate cortex, right subgenual cingulate cortex, and right PCC compared to ARMS who did not make the transition to psychosis (ARMS-NT) (Röthlisberger et al., 2012).

Cannabis use and abuse occurs much more frequently in psychosis patients than in the general population (Koskinen et al., 2010) and is suspected to be a risk factor for psychosis (Drewe et al., 2004; Moore et al., 2007). Neurobiological models for this association postulate that delta-9- 
tetrahydrocannabinol (THC, the main psychoactive component of cannabis) causes

dopaminergic imbalances by increasing the dopaminergic tone in striatal regions of the brain via activation of cannabinoid 1 (CB1) receptors and decreasing dopamine levels in prefrontal regions of the brain (Kuepper et al., 2010). However, most evidence supporting this stems from animal research (Kuepper et al., 2010) and has not been empirically investigated in humans. Nevertheless, there is clear evidence that there are associations between schizophrenia and alterations of dopamine (for review, see Howes and Kapur, 2009) as well as between schizophrenia and brain structural alterations (for review, see Steen et al., 2006). Another neurobiological explanation for the cannabis-psychosis association could be that cannabis contributes to abnormalities in brain structure and therefore the development of psychotic symptoms. Although studies investigating how cannabis affects brain structure of healthy individuals have produced inconsistent results, the most consistent brain volume abnormalities associated with cannabis use in healthy controls were found in medial temporal regions (for review, see Lorenzetti et al., 2010; Martin-Santos et al., 2010). It has been reported that in ACC, cannabis may affect the integrity of white matter fiber tracts (Gruber and YurgelunTodd, 2005). Functional MRI studies found that acute administration of THC or marijuana increases resting activity and activation of the ACC during cognitive tasks (Martin-Santos et al., 2010). There is only one structural MRI study with healthy controls that took into account the effects of cannabis on the cingulum volume. Specifically, Cousijin et al. (2012) reported negative associations between amygdala/ hippocampus grey matter volume and amount of cannabis use but no associations of cannabis with ACC and striatum in a voxel based morphometry study (Cousijn et al., 2012).

In contrast to humans, animal studies have demonstrated that THC induced dose-dependent neurotoxic changes in brain regions rich in CB1 receptors (Downer et al., 2001; Landfield et al., 1988; Whitaker-Azmitia et al., 2000). However, this could also be due to the fact that in animal 
studies, the THC doses administered were high and THC was often not mixed with other cannabinoids. There are also studies which reported neuroprotective effects of cannabinoids in animals (e.g. Sagredo et al., 2011).

We have recently reviewed studies comparing brain volumes of cannabis using patients with psychosis with those of non-using psychosis patients and healthy controls (Rapp et al., 2012). The systematic review demonstrated that cannabis use is associated with smaller volume of global and specific brain structures, particularly in CB1 receptor rich brain regions, such as the cingulate cortex (Bangalore et al., 2008; Szeszko et al., 2007), prefrontal cortices (James et al., 2011; Rais et al., 2010) and the cerebellum (James et al., 2011; Solowij et al., 2011). Furthermore, the associations between brain structural volume reductions and cannabis consumption were more pronounced in psychosis patients and individuals at genetic risk for psychosis than in healthy controls, suggesting that these groups might be particularly vulnerable to brain volume loss due to cannabis exposure. Our literature review also revealed that, to date, only one study (Stone et al., 2011) investigated the effect of cannabis on brain structure in ARMS. By analysing the interaction between the two diagnostic groups (ARMS / first episode psychosis (FEP)) and cannabis use, conclusions can be drawn as to whether cannabis use is associated with brain structure in a disease stage-dependent manner. To this end, associations between cannabis consumption and cingulum volume in both ARMS and FEP samples that were obtained within the same study, were investigated. We hypothesized that:

1. cannabis use is associated with lower grey matter volumes in the cingulate cortex in both diagnostic groups.

2. these associations are more pronounced in FEP than in ARMS subjects. 


\section{Methods}

\subsection{Setting and recruitment}

This study was part of the Basel FePsy (Früherkennung von Psychosen) study, which aims to improve the early detection of psychosis. The FePsy study has been described in detail elsewhere (Riecher-Rössler et al., 2007; Riecher-Rössler et al., 2009). Subjects were recruited into the study via a specialised outpatient clinic at the Psychiatric Outpatient Department at the University Hospital Basel. This clinic was set up specifically to identify, assess, and follow up individuals in the early stages of psychosis. The study was approved by the Ethics Committee of Basel, Switzerland (EKBB), and written informed consent was obtained from each of the participants.

\subsection{Screening procedure}

For screening, the Basel Screening Instrument for Psychosis (BSIP) was used (Riecher-Rössler et al., 2008). Individuals were assessed and identified as ARMS, FEP, or "not at risk for psychosis" (i. e. other psychiatric diseases) subjects.

\subsection{Participants}

In this study, we present data of 60 patients from the Basel FePsy study who agreed to participate in the imaging arm of the study. 23 of the patients were identified as FEP and 37 as ARMS subjects. Data of this study are overlapping with our previous studies (Bühlmann et al., 2010; Röthlisberger et al., 2012; Walter et al., 2012) (overlapping $n=60$ ).

\subsection{Cannabis, alcohol and other drug use}

Cannabis, alcohol and other drug use was determined both for ARMS and FEP at study inclusion by using the Basel Interview for Psychosis (BIP) (Riecher-Rössler et al., in 
preparation), a structured and specifically developed interview for the assessment of psychosis development, which is much more detailed than the BSIP. The BIP contains two items assessing the frequency of past and present cannabis/alcohol/other drug consumption. Past drug consumption refers to lifetime consumption, present consumption to the frequency participants reported to usually use cannabis during assessments. Frequency of substance use is assessed by these items on a five-point ordinal scale using the following response categories: daily, several times a week, several times a month, less than several times a month, and not at all. For the present analyses, only current substance use was considered. A dichotomous variable of cannabis use was created differentiating between current cannabis users (= daily, several times a week, several times a month and less than several times a month) and non-users (= no use at all).

In $60 \%$ of the included patients, cannabis use was additionally assessed by urine toxicology screens. Urine tests were considered positive when $\mathrm{THC}-\mathrm{COOH}$ was present in the urine in a concentration of at least $10 \mu \mathrm{g} / \mathrm{l}$, in order to infer a detection window of $\approx 1$ month. Although urine tests were only available in a subset of our sample, the agreement between urine tests and the questionnaire item on current use was excellent. That is, all patients with cannabis-positive urine had responded to the questionnaire item measuring current cannabis use with a frequency of at least rarely and all patients with cannabis-negative urine had responded with a frequency less than several times per month. Hence, relying only on information of the BIP in those patients who did not have urine toxicology screens was considered well justified.

\subsection{Structural MRI}

\subsubsection{Image acquisition}

Subjects were scanned using a Siemens (Erlangen, Germany) Magnetom Vision 1.5 T scanner at the University Hospital Basel. Head movement was minimized by foam padding and velcro 
straps across the forehead and chin. A three-dimensional volumetric spoiled gradient recalled echo sequence generated 176 contiguous, $1 \mathrm{~mm}$ thick sagittal slices. Imaging parameters were: time-to-echo, $4 \mathrm{msec}$; time-to-repetition, $9.7 \mathrm{msec}$; flip angle, 12; matrix size, $200 \times 256$; field of view, 25.6 × $25.6 \mathrm{~cm}$ matrix; voxel dimensions, $1.28 \times 1 \times 1 \mathrm{~mm}$.

\subsubsection{Manual segmentation of cingulum and whole brain volume}

Original DICOM data were converted to Analyze 7.5 format in axial direction coded to ensure blindness of the investigator and patients' confidentiality and finally imported into the imageanalysis software AMIRA (Kappos et al., 2006). Anatomical definition of the ACC and PCC were made by using Talairach CoPlanar Stereotaxic Atlas (Talairach et al., 1993). The paracingulate gyrus was considered to be a part of ACC, which is in accordance with several other studies (e.g. Koo et al., 2008). The cingulum volume was traced strictly following the protocol of Röthlisberger et al. (2012) (see online supplementary document 1). In the present study, we focused upon the anterior (left/right) and the posterior (left/right) cingulum.

The whole brain volume (WBV) was measured to correct for differences in head size as previously described (Borgwardt, McGuire et al. 2007; Borgwardt, Riecher-Rössler et al. 2007; Haller, Borgwardt et al. 2009).

\subsubsection{Intra-rater reliability / inter-rater reliability}

To assess an intra-rater reliability, a manual segmentation in ten consecutive cases was accomplished by the same person (MR) within two weeks. The intra-rater reliability was as follows: $I C C_{\text {total left }}=0.95, I C C_{\text {total right }}=0.95, I C C_{\text {ant left }}=0.98, I C C_{\text {ant right }}=0.98, I C C_{\text {post left }}=0.81$, $\mathrm{ICC}_{\text {post right }}=0.89$.

In order to calculate an inter-rater reliability, a second rater (AW) manually traced the cingulum of ten randomly selected cases. The inter-rater reliability was as follows: $I C C_{\text {total left }}=0.96, I C C_{\text {total }}$ right $=0.97, I C C_{\text {ant left }}=0.97, I C C_{\text {ant right }}=0.98, I C C_{\text {post left }}=0.95, I C C_{\text {post right }}=0.94$. 
The researchers (MR and AW) who traced the cingulate volumes were blind to the group status at any time of the study. They were trained and supervised by a specialised neuroradiologist according to the procedure described in online supplementary document 1 . Once the gray matter of the cingulate cortex had been traced, volumes (in mm3) were calculated by computing the number of voxels from each traced image.

\subsection{Statistical analysis}

All analyses were performed using the Statistical Package for the Social Sciences (SPSS) version 19. Two tailed $t$-tests for the continuous and $\chi^{2}$ tests for the categorical variables were used in order to compare groups for demographic and clinical data $(p<0.05)$.

A repeated measures ANCOVA model was calculated including grey matter volume as dependent variable, hemisphere (left/right) and region (anterior/posterior cingulum) as within subjects variables and group (ARMS/FEP) and cannabis use (yes/no) as between subject variables. Age, sex, medication, whole brain volume and alcohol use were entered as covariates $(p<0.05)$

\section{Results}

\subsection{Sample characteristics}

The socio-demographic characteristics of the sample are presented in Table 1. There were no significant differences between ARMS and FEP subjects with regard to age, gender, education, alcohol use, and whole brain volume. FEP subjects had a significantly higher BPRS total score ( $t$ $(56)=-4.33, p<0.001)$ and showed a statistical trend toward a higher SANS total score $(t(57)=$ $-1.71, p=0.09$ ) than ARMS subjects. A large proportion of FEP subjects were scanned within 13 days of first contact. Therefore, most of the FEP subjects (14/23) were also antipsychotic- 
naïve. The remaining nine participants were prescribed second generation antipsychotic medications (six of them for less than one month; three participants received risperidone, six olanzapine). Thirty-three out of 37 ARMS subjects had never received antipsychotic medication. Four ARMS subjects had received low doses of second generation antipsychotic medication during no more than three weeks for behavioural control by the referring psychiatrist or general practitioner prior to study inclusion. Three of these participants had received olanzapine and one risperidone.

\subsection{Cannabis use}

Of the ARMS subjects, $63.3 \%$ reported to currently use cannabis with a frequency ranging from daily to less than monthly. From the FEP subjects, $75 \%$ were current cannabis users. ARMS and FEP subjects did not differ with regards to frequency of cannabis use. Current cannabis users and non-users did not differ with regard to age, gender, medication, alcohol, whole brain volume, BPRS total, and SANS total scores. However, cannabis users had a significantly lower education $(t(58)=3.04, p=0.004)$ than non-users.

\section{Insert Table 1 here}

\subsection{Brain volumes in cannabis users and non-users}

Figure 1 shows the means \pm one standard deviations of cingulum volumes for cannabis users and non-users in the two diagnostic groups (ARMS/FEP).

Insert Figure 1 here

The within subject contrasts of the ANCOVA including hemisphere (left/right) and region (ACC/PCC) as within subjects factors and group (ARMS/FEP) and cannabis use (yes/no) as 
between subject factors with age, sex, medication, whole brain volume and alcohol use as covariates revealed a significant three way interaction between region, hemisphere and cannabis use $\left(F(1,51)=5.62\right.$, Partial $\left.\eta^{2}=0.10, p=0.022\right)$. To further examine this effect, each region was analysed in separate ANCOVA models using hemisphere as within subject factor, group and cannabis use as between subject factors and age, sex, medication, whole brain volumes, and alcohol use as covariates (Table 2). The results revealed that there was a significant negative effect of cannabis use on $\operatorname{PCC}\left(F(1,51)=8.96\right.$, Partial $\left.\eta^{2}=0.15, p=0.004\right)$ which was independent of diagnostic group and hemisphere and a statistical trend for an interaction effect between hemisphere and cannabis use $\left(F(1,51)=3.95\right.$, Partial $\eta^{2}=0.07, p=$ 0.052) on the ACC which was independent of group. Two further post hoc univariate ANCOVAs for each of the two hemispheres revealed a significant effect of cannabis use only on the left $\operatorname{ACC}\left(F(1,51)=7.02\right.$, Partial $\left.\eta^{2}=0.12, p=0.011\right)$.

When the analyses were repeated by excluding individuals using other illicit drugs (i.e., one using MDMA and one using psychedelics), the results did not change.

\author{
Insert Table 2 here
}

\title{
4. Discussion
}

This study investigated whether cannabis use is associated with cingulate cortex volume in early stages of psychosis. As the first study in this field, we included both an ARMS and FEP sample. We found a significant negative effect of cannabis use on PCC volumes which was independent of the hemisphere and diagnostic group and all other covariates we controlled for. In the ACC, we found a significant negative effect only on left hemisphere, which was again independent of 
diagnostic group. The findings remained the same when individuals using other illicit drugs were excluded.

\subsection{Prevalence of cannabis use}

$63.3 \%$ of our ARMS and $75 \%$ of the FEP subjects reported to currently use cannabis (Table 1 ). According to a Swiss health survey from the year $2004,13.3 \%$ of all $13-29$ year aged individuals stated to currently use cannabis (Arbeitsgruppe Cannabismonitoring, 2005). Therefore, the prevalence of cannabis use in our FEP/ARMS sample seems to be much higher than in the general population which is in line with other studies (Drewe et al., 2004; Koskinen et al., 2010). Cannabis users in our sample had significantly less educational years than non-users. Similarly, other studies investigating non-help seeking subjects reported that cannabis use is associated with reduced educational achievement (Fergusson and Boden, 2008; Patton et al., 2007), increased welfare dependence (Schmidt et al., 1998) and reduced income (Degenhardt et al., 2007), however it is often not clear whether cannabis consumption actually preceded this (Barnwell et al., 2006).

\subsection{Integration of other studies on brain structure in cannabis users with psychosis}

Our reported associations of cannabis use with reduced brain volume are in line with previous MRI studies: within a recent review from our group (Rapp et al., 2012) in which 15 in vivo structural MRI studies were included, 11 found that cannabis use was associated with a decrease in global or specific brain structures in psychosis patients (Bangalore et al., 2008; Habets et al., 2011; Ho et al., 2011; James et al., 2011; Rais et al., 2008; Rais et al., 2010; Solowij et al., 2011; Szeszko et al., 2007) and in individuals at clinical/genetic risk for psychosis (Habets et al., 2011; Stone et al., 2011; Welch et al., 2011a; Welch et al., 2011b). The cingulate cortex was specifically investigated in two studies (Bangalore et al., 2008; Szeszko et al., 2007). 
Szeszko et al. (2007) compared frontal gyrus, anterior cingulate gyrus, and orbital frontal lobe in 20 FEP subjects with an additional diagnosis of cannabis abuse or dependence with 31 FEP subjects with no cannabis use and with healthy volunteers. They reported that patients experiencing a first episode of schizophrenia who have a history of cannabis use have less whole anterior and specifically left anterior cingulate cortex grey matter compared with similar patients who do not use cannabis and with healthy volunteers. In a similar study, Bangalore et al. (2008) investigated the dorsolateral prefrontal cortex, hippocampus, posterior cingulate, and cerebellum in 57 FEP subjects both with and without comorbid cannabis use. A decrease in grey matter density in the right posterior cingulate cortex (PCC) was found in cannabis using subjects when compared with non-using subjects and healthy controls.

\subsection{The cingulate cortex, its involvement in psychosis and vulnerability to the effects of} cannabis use

The findings from Bangalore et al. (2008) and Szeszko et al. (2007) and our present results suggest that the cingulate cortex might be a brain region specifically vulnerable for the influence of cannabis use in psychosis. As indicated by recent studies, a decrease of volume in this brain region might be a specific risk factor for transition to psychosis. More precisely, anterior subunits have been shown to be affected in early stages during the prodrome, posterior subunits in later stages when it comes to a transition to psychosis (Borgwardt et al., 2008; Fornito et al., 2008; Röthlisberger et al., 2012).

The cingulum is a brain area rich in CB1 receptors (Eggan and Lewis, 2007), which are responsible for the psychotropic actions of cannabis. CB1 receptors deliver the effects of both endogenous and exogenous (such as THC) cannabinoids from cannabis sativa in the central nervous system and are therefore part of the endocannabinoid system (Kuepper et al., 2010). Post mortem studies (Dean et al., 2001; Eggan et al., 2008) revealed that schizophrenia is 
associated with alterations in the endocannabinoid system. Zavitsanou et al. (2004) examined post-mortem radioligand binding of $[3 \mathrm{H}] \mathrm{SR} 141716 \mathrm{~A}$, an antagonist that specifically targets $\mathrm{CB} 1$ receptors of the endogenous cannabinoid system, on ACC sections using quantitative autoradiography. They found a significant $64 \%$ increase in $\left[{ }^{3} \mathrm{H}\right] \mathrm{SR} 141716 \mathrm{~A}$ specific binding to CB1 receptors in the schizophrenia group compared to a control group. Dean et al. (2001) additionally showed that acute cannabis effects in both patients with schizophrenia and healthy controls is associated with change in density of CB1 in tissue. Therefore, a plausible consequence of chronic cannabis use might be that structures rich in CB1 receptors, such as the subunits of the cingulate cortex, decrease in volume and therefore might accelerate the progression of psychosis. As described before, the left anterior subunit might be a specific marker for ARMS, and the posterior subunit a specific marker for transition to psychosis (ARMST, FEP) (Röthlisberger et al., 2012).This could be a consequence of CB1 receptors deferring from protecting the brain against excitotoxic influence (Rais et al., 2010). CB1 receptors activated by the endogenous cannabinoids protect the brain from excitotoxic events (Kim et al., 2006), such as N-methyl-Daspartate (NMDA) toxicity (Kim et al., 2006) or kainic acid injection (Marsicano et al., 2003). This physiological process of CB1 receptors playing a neuroprotective role against excitotoxic events might be disrupted by exogenous cannabinoids (Egerton et al., 2006).

A possible influence of cannabis on ACC also seems plausible as it plays an important role in normal cognition and attention (Bush et al., 2000). Long term cannabis use has been reported to impair those functions (e.g. Crean et al., 2011; Solowij et al., 2002) which also resemble core negative symptoms in psychosis, although this effect was not consistently confirmed in the literature (e.g. Fried et al., 2005; Grant et al., 2003). Additionally, changes in dopaminergic, serotonergic, glutamatergic and GABAergic systems were reported in ACC in schizophrenia 
which are all interconnected with the cannabinoid system (Zavitsanou and Huang, 2002; Zavitsanou et al., 2002).

In our study, we specifically found strong associations of cannabis use with PCC, which was similarly reported by Bangalore et al. (2008). It has been shown that poor outcome in schizophrenia is associated with smaller PCC (Mitelman et al., 2005), and reductions in the volume of bilateral PCC seem to be predictive for transition to psychosis in ARMS subjects (Pantelis et al., 2003; Röthlisberger et al., 2012).

\subsection{Brain changes associated with cannabis use in individuals at risk for psychosis} To date, studies of subjects at high genetic (Habets et al., 2011; Welch et al., 2011a; Welch et al., 2011b) or clinical (Stone et al., 2011) risk for psychosis found that cannabis use was associated with altered global and specific grey matter volume, and three of them also reported that this effect was stronger in individuals at risk for psychosis than in healthy controls, suggesting that individuals at risk for psychosis may be more vulnerable for the neurotoxic effect of cannabis than healthy individuals. Welch et al. (2011a) who investigated the association between substance misuse and brain abnormality not only found that use of cannabis by people at genetic high risk for schizophrenia is associated with brain abnormalities but also that individuals at risk for psychosis consuming cannabis had an elevated rate of developing schizophrenia than those subjects without cannabis use.

These previous findings are in line with the present data which showed that cannabis using ARMS subjects had smaller cingulum volume than non-users.

We a priori expected an interaction between group status (ARMS/FEP) and cannabis use such that there is more grey matter reduction in consequence of cannabis use in FEP than in ARMS subjects. However, this hypothesis could not be confirmed within our study, suggesting that the brain in psychosis is affected by cannabis use regardless of the disease stage. 


\subsection{Limitations}

The present study has several limitations: first, we could not compare the findings with a cannabis using healthy control sample. Hence, we were unable to analyse whether cannabis has a differential effect on brain structure in psychosis patients compared to healthy individuals. Second, we only assessed the frequency of current cannabis use but not a cumulative dose of THC content. Therefore, associations between cumulative dose of cannabis and cingulum volume could not be examined. Finally, given the cross-sectional nature of this study, the association between cannabis use and cingulum volume cannot be interpreted causally..

\subsection{Conclusions}

Our hypotheses could be partly confirmed: there were strong associations of cannabis use with the cingulate cortex, a region identified as being rich in CB1 receptors. However, this effect was not different for FEP and ARMS subjects. This might suggest that both ARMS as well as FEP subjects could be particularly sensitive to exogenous activation of these specific receptors.

\section{Acknowledgements}

We thank Prof. Ernst-Wilhelm Radue (Medical Image Analysis Centre Basel) for supervising the tracing process. We also kindly thank Claudine Pfister/Martina Klemm for their help in preparing the manuscript.

This project was supported by grants of the Swiss National Science Foundation (no. 3200057216.99, no. 3200-057216.99 and no. PBBSB-106936) and the Nora van Meeuwen-Haefliger Stiftung, Basel $(\mathrm{CH})$. 


\section{References}

Arbeitsgruppe Cannabismonitoring, 2005. Cannabiskonsum in der Schweiz und die Konsequenzen - Ein aktueller Überblick 2004. Bundesamt für Gesundheit. http://www.bag.admin.ch/themen/drogen/00042/00643/00646/02287/index.html?lang=de\& download=M3wBPgDB/8ull6Du36WcnojN14in3qSbnpWVZW2YIE6p1rJgsYfhyt3NhqbdqIV +baqwbKbXrZ6lhuDZz8mMps2go6fo.

Bangalore, S.S., Prasad, K.M.R., Montrose, D.M., Goradia, D.D., Diwadkar, V.A., Keshavan, M.S., 2008. Cannabis use and brain structural alterations in first episode schizophrenia A region of interest, voxel based morphometric study. Schizophrenia Research 99, 1-6.

Barnwell, S., Earleywine, M., Wilcox, R., 2006. Cannabis, motivation, and life satisfaction in an internet sample. Substance Abuse Treatment, Prevention, and Policy 1, 2.

Borgwardt, S.J., Dickey, C., Pol, H.H., Whitford, T.J., DeLisi, L.E., 2009. Workshop on defining the significance of progressive brain change in schizophrenia: December 12, 2008 American College of Neuropsychopharmacology (ACNP) all-day satellite, Scottsdale, Arizona The rapporteurs' report. Schizophrenia Research 112, 32-45.

Borgwardt, S.J., McGuire, P., Fusar-Poli, P., Radue, E.-W., Riecher-Rössler, A., 2008. Anterior cingulate pathology in the prodromal stage of schizophrenia. Neuroimage 39, 553-554.

Bühlmann, E., Berger, G.E., Aston, J., Gschwandtner, U., Pflueger, M.O., Borgwardt, S.J., Radue, E.W., Riecher-Rössler, A., 2010. Hippocampus abnormalities in at risk mental states for psychosis? A cross-sectional high resolution region of interest magnetic resonance imaging study. Journal of Psychiatric Research 44, 447-453.

Bush, G., Luu, P., Posner, M.I., 2000. Cognitive and emotional influences in anterior cingulate cortex. Trends Cognitive Science 4, 215-222.

Cousijn, J., Wiers, R.W., Ridderinkhof, K.R., van den Brink, W., Veltman, D.J., Goudriaan, A.E., 2012. Grey matter alterations associated with cannabis use: Results of a VBM study in heavy cannabis users and healthy controls. Neuroimage 59, 3845-3851.

Crean, R.D., Crane, N.A., Mason, B.J., 2011. An evidence based review of acute and long-term effects of cannabis use on executive cognitive functions. Journal of Addiction Medicine 5, $1-8$.

Dean, B., Sundram, S., Bradbury, R., Scarr, E., Copolov, D., 2001. Studies on [H-3]CP-55940 binding in the human central nervous system: Regional specific changes in density of cannabinoid-1 receptors associated with schizophrenia and cannabis use. Neuroscience 103, 9-15.

Degenhardt, L., Chiu, W.T., Sampson, N., Kessler, R.C., Anthony, J.C., 2007. Epidemiological patterns of extra-medical drug use in the United States: Evidence from the national Comorbidity Survey Replication, 2001-2003. Drug and Alcohol Dependence 90, 210-223.

Downer, E., Boland, B., Fogarty, M., Campbell, V., 2001. [DELTA]9-Tetrahydrocannabinol induces the apoptotic pathway in cultured cortical neurones via activation of the CB1 receptor. NeuroReport 12, 3973-3978.

Drewe, M., Drewe, J., Riecher-Rössler, A., 2004. Cannabis and risk of psychosis. Swiss Medical Weekly 134, 659-663.

Egerton, A., Allison, C., Brett, R.R., Pratt, J.A., 2006. Cannabinoids and prefrontal cortical function: Insights from preclinical studies. Neuroscience and Biobehavioral Reviews 30, 680-695.

Eggan, S.M., Hashimoto, T., Lewis, D.A., 2008. Reduced cortical cannabinoid 1 receptor messenger RNA and protein expression in schizophrenia. Archives of General Psychiatry $65,772-784$. 
Eggan, S.M., Lewis, D.A., 2007. Immunocytochemical distribution of the cannabinoid CB1 receptor in the primate neocortex: a regional and laminar analysis. Cerebral Cortex 17, 175-191.

Fergusson, D.M., Boden, J.M., 2008. Cannabis use and later life outcomes. Addiction 103, 969976.

Fornito, A., Yung, A.R., Wood, S.J., Phillips, L.J., Nelson, B., Cotton, S., Velakoulis, D., McGorry, P.D., Pantelis, C., Yucel, M., 2008. Anatomic abnormalities of the anterior cingulate cortex before psychosis onset: An MRI study of ultra-high-risk individuals. Biological Psychiatry 64, 758-765.

Fried, P.A., Watkinson, B., Gray, R., 2005. Neurocognitive consequences of marihuana--a comparison with pre-drug performance. Neurotoxicology and Teratology 27, 231-239.

Fusar-Poli, P., Allen, P., McGuire, P., 2008. Neuroimaging studies of the early stages of psychosis: a critical review. European Psychiatry 23, 237-244.

Fusar-Poli, P., Borgwardt, S., Crescini, A., Deste, G., Kempton, M.J., Lawrie, S., Mc Guire, P., Sacchetti, E., 2011. Neuroanatomy of vulnerability to psychosis: a voxel-based metaanalysis. Neuroscience Biobehavioral Review 35, 1175-1185.

Grant, I., Gonzalez, R., Carey, C.L., Natarajan, L., Wolfson, T., 2003. Non-acute (residual) neurocognitive effects of cannabis use: a meta-analytic study. Journal of the International Neuropsychological Society 9, 679-689.

Gruber, S.A., Yurgelun-Todd, D.A., 2005. Neuroimaging of marijuana smokers during inhibitory processing: a pilot investigation. Cognitive Brain Research 23, 107-118.

Habets, P., Marcelis, M., Gronenschild, E., Drukker, M., van Os, J., 2011. Reduced Cortical Thickness as an Outcome of Differential Sensitivity to Environmental Risks in Schizophrenia. Biological Psychiatry 69, 487-494.

Ho, B.C., Wassink, T.H., Ziebell, S., Andreasen, N.C., 2011. Cannabinoid receptor 1 gene polymorphisms and marijuana misuse interactions on white matter and cognitive deficits in schizophrenia. Schizophrenia Research 128, 66-75.

Howes, O.D., Kapur, S., 2009. The dopamine hypothesis of schizophrenia: version III-the final common pathway. Schizophrenia Bulletin 35, 549-562.

James, A., Hough, M., James, S., Winmill, L., Burge, L., Nijhawan, S., Matthews, P.M., Zarei, M., 2011. Greater white and grey matter changes associated with early cannabis use in adolescent-onset schizophrenia (AOS). Schizophrenia Research 128, 91-97.

Kappos, L., Antel, J., Comi, G., Montalban, X., O'Connor, P., Polman, C.H., Haas, T., Korn, A.A., Karlsson, G., Radue, E.W., 2006. Oral fingolimod (FTY720) for relapsing multiple sclerosis. New England Journal of Medicine 355, 1124-1140.

Kim, S.H., Won, S.J., Mao, X.O., Jin, K.L., Greenberg, D.A., 2006. Molecular mechanisms of cannabinoid protection from neuronal excitotoxicity. Molecular Pharmacology 69, 691-696.

Koo, M.S., Levitt, J.J., Salisbury, D.F., Nakamura, M., Shenton, M.E., McCarley, R.W., 2008. A cross-sectional and longitudinal magnetic resonance imaging study of cingulate gyrus gray matter volume abnormalities in first-episode schizophrenia and first-episode affective psychosis. Archives of General Psychiatry 65, 746-760.

Kopelman, A., Andreasen, N.C., Nopoulos, P., 2005. Morphology of the anterior cingulate gyrus in patients with schizophrenia: Relationship to typical neuroleptic exposure. American Journal of Psychiatry 162, 1872-1878.

Koskinen, J., Lohonen, J., Koponen, H., Isohanni, M., Miettunen, J., 2010. Rate of cannabis use disorders in clinical samples of patients with schizophrenia: a meta-analysis. Schizophrenia Bulletin 36, 1115-1130.

Kuepper, R., Morrison, P.D., van Os, J., Murray, R.M., Kenis, G., Henquet, C., 2010. Does dopamine mediate the psychosis-inducing effects of cannabis? A review and integration of findings across disciplines. Schizophrenia Research 121, 107-117. 
Landfield, P.W., Cadwallader, L.B., Vinsant, S., 1988. Quantitative changes in hippocampal structure following long-term exposure to $\Delta 9$-tetrahydrocannabinol: possible mediation by glucocorticoid systems. Brain Research 443, 47-62.

Lorenzetti, V., Lubman, D.I., Whittle, S., Solowij, N., Yücel, M., 2010. Structural MRI Findings in Long-Term Cannabis Users: What Do We Know? Substance Use \& Misuse 45, 17871808.

Marsicano, G., Goodenough, S., Monory, K., Hermann, H., Eder, M., Cannich, A., Azad, S.C., Cascio, M.G., Gutierrez, S.O., van der Stelt, M., Lopez-Rodriguez, M.L., Casanova, E., Schutz, G., Zieglgansberger, W., Di Marzo, V., Behl, C., Lutz, B., 2003. CB1 cannabinoid receptors and on-demand defense against excitotoxicity. Science 302, 84-88.

Martin-Santos, R., Fagundo, A.B., Crippa, J.A., Atakan, Z., Bhattacharyya, S., Allen, P., FusarPoli, P., Borgwardt, S., Seal, M., Busatto, G.F., McGuire, P., 2010. Neuroimaging in cannabis use: a systematic review of the literature. Psychological Medicine 40, 383-398.

Mitelman, S.A., Shihabuddin, L., Brickman, A.M., Hazlett, E.A., Buchsbaum, M.S., 2005. Volume of the cingulate and outcome in schizophrenia. Schizophrenia Research 72, 91-108.

Moore, T.H., Zammit, S., Lingford-Hughes, A., Barnes, T.R., Jones, P.B., Burke, M., Lewis, G., 2007. Cannabis use and risk of psychotic or affective mental health outcomes: a systematic review. Lancet 370, 319-328.

Pantelis, C., Velakoulis, D., McGorry, P.D., Wood, S.J., Suckling, J., Phillips, L.J., Yung, A.R., Bullmore, E.T., Brewer, W., Soulsby, B., Desmond, P., McGuire, P.K., 2003. Neuroanatomical abnormalities before and after onset of psychosis: a cross-sectional and longitudinal MRI comparison. Lancet 361, 281-288.

Patton, G.C., Coffey, C., Lynskey, M.T., Reid, S., Hemphill, S., Carlin, J.B., Hall, W., 2007. Trajectories of adolescent alcohol and cannabis use into young adulthood. Addiction 102, 607-615.

Rais, M., Cahn, W., Van Haren, N., Schnack, H., Caspers, E., Pol, H.H., Kahn, R., 2008. Excessive brain volume loss over time in cannabis-using first-episode schizophrenia patients. American Journal of Psychiatry 165, 490-496.

Rais, M., van Haren, N.E.M., Cahn, W., Schnack, H.G., Lepage, C., Collins, L., Evans, A.C., Pol, H.E.H., Kahn, R.S., 2010. Cannabis use and progressive cortical thickness loss in areas rich in CB1 receptors during the first five years of schizophrenia. European Neuropsychopharmacology 20, 855-865.

Rapp, C., Bugra, H., Riecher-Rössler, A., Tamagni, C., Borgwardt, S., 2012. Effects of cannabis use on human brain structure in psychosis: a systematic review combining in vivo structural neuroimaging and post mortem studies. Current Pharmaceutical Design 18, 5070-5080.

Riecher-Rössler, A., Aston, J., Ventura, J., Merlo, M., Borgwardt, S., Gschwandtner, U., Stieglitz, R.D., 2008. The Basel screening instrument for psychosis (BSIP): development, structure, reliability and validity. Fortschritte Neurologie Psychiatrie 76, 207-216.

Riecher-Rössler, A., Gschwandtner, U., Aston, J., Borgwardt, S., Drewe, M., Fuhr, P., Pflüger, M., Radu, W., Schindler, C., Stieglitz, R.D., 2007. The Basel early-detection-of-psychosis (FEPSY)-study - design and preliminary results. Acta Psychiatrica Scandinavica 115, 114125.

Riecher-Rössler, A., Pflueger, M.O., Aston, J., Borgwardt, S.J., Brewer, W.J., Gschwandtner, U., Stieglitz, R.D., 2009. Efficacy of using cognitive status in predicting psychosis: a 7-year follow-up. Biological Psychiatry 66, 1023-1030.

Röthlisberger, M., Riecher-Rossler, A., Aston, J., Fusar-Poli, P., Radu, E.-W., Borgwardt, S., 2012. Cingulate volume abnormalities in emerging psychosis. Current Pharmaceutical Design 18, 495-504. 
Sagredo, O., Pazos, M.R., Satta, V., Ramos, J.A., Pertwee, R.G., Fernandez-Ruiz, J., 2011. Neuroprotective effects of phytocannabinoid-based medicines in experimental models of Huntington's disease. Journal of Neuroscience Research 89, 1509-1518.

Schmidt, L., Weisner, C., Wiley, J., 1998. Substance abuse and the course of welfare dependency. American Journal of Public Health 88, 1616-1622.

Smieskova, R., Fusar-Poli, P., Allen, P., Bendfeldt, K., Stieglitz, R.D., Drewe, J., Radue, E.W., McGuire, P.K., Riecher-Rössler, A., Borgwardt, S.J., 2010. Neuroimaging predictors of transition to psychosis A systematic review and meta-analysis. Neuroscience and Biobehavioral Reviews 34, 1207-1222.

Solowij, N., Stephens, R.S., Roffman, R.A., Babor, T., Kadden, R., Miller, M., Christiansen, K., McRee, B., Vendetti, J., Res, M.T.P., 2002. Cognitive functioning of long-term heavy cannabis users seeking treatment. Journal of the American Medical Association 287, 1123-1131.

Solowij, N., Yucel, M., Respondek, C., Whittle, S., Lindsay, E., Pantelis, C., Lubman, D.I., 2011. Cerebellar white-matter changes in cannabis users with and without schizophrenia. Psychological Medicine 41, 2349-2359.

Steen, R.G., Mull, C., McClure, R., Hamer, R.M., Lieberman, J.A., 2006. Brain volume in firstepisode schizophrenia - Systematic review and meta-analysis of magnetic resonance imaging studies. British Journal of Psychiatry 188, 510-518.

Stone, J.M., Bhattacharyya, S., Barker, G., McGuire, P.K., 2011. Substance use and regional grey matter volume in individuals at high risk of psychosis. European Neuropsychopharmacology 21, S63-S64.

Szeszko, P.R., Robinson, D.G., Sevy, S., Kumra, S., Rupp, C.I., Betensky, J.D., Lencz, T., Ashtari, M., Kane, J.M., Malhotra, A.K., Gunduz-Bruce, H., Napolitano, B., Bilder, R.M., 2007. Anterior cingulate grey-matter deficits and cannabis use in first-episode schizophrenia. British Journal of Psychiatry 190, 230-236.

Talairach, J., Missir, O., Tournoux, P., 1993. Referentially oriented cerebral MRI anatomy: an atlas of stereotaxic anatomical correlations for gray and white matter. Thieme Medical Publishers, New York.

Vogt, B.A., Finch, D.M., Olson, C.R., 1992. Functional heterogeneity in cingulate cortex: the anterior executive and posterior evaluative regions. Cerebral Cortex 2, 435-443.

Vogt, B.A., Laureys, S., 2005. Posterior cingulate, precuneal and retrosplenial cortices: cytology and components of the neural network correlates of consciousness. Progress in Brain Research 150, 205-217.

Vogt, B.A., Vogt, L., Laureys, S., 2006. Cytology and functionally correlated circuits of human posterior cingulate areas. Neuroimage 29, 452-466.

Walter, A., Studerus, E., Smieskova, R., Kuster, P., Aston, J., Lang, U.E., Radue, E.-W., Riecher-Rössler, A., Borgwardt, S., 2012. Hippocampal volume in subjects at high risk of psychosis: A longitudinal MRI study. Schizophrenia Research 142, 217-222.

Welch, K.A., Mclntosh, A., Moorhead, W., Whalley, H., Job, D., Owens, D., Lawrie, S., Johnstone, E., 2011a. The impact of substance use on brain structure in people at genetically high risk of schizophrenia. Schizophrenia Bulletin 37, 182-182.

Welch, K.A., Stanfield, A.C., Mclntosh, A.M., Whalley, H.C., Job, D.E., Moorhead, T.W., Owens, D.G., Lawrie, S.M., Johnstone, E.C., 2011b. Impact of cannabis use on thalamic volume in people at familial high risk of schizophrenia. British Journal of Psychiatry.

Whitaker-Azmitia, P.M., Lawston, J., Borella, A., Robinson, J.K., 2000. Changes in hippocampal morphology following chronic treatment with the synthetic cannabinoid WIN 55,212-2. Brain Research 877, 407-410.

Yung, A.R., Yuen, H.P., McGorry, P.D., Phillips, L.J., Kelly, D., Dell'Olio, M., Francey, S.M., Cosgrave, E.M., Killackey, E., Stanford, C., Godfrey, K., Buckby, J., 2005. Mapping the 
onset of psychosis: The comprehensive assessment of at-risk mental states. Australian and New Zealand Journal of Psychiatry 39, 964-971.

Zavitsanou, K., Garrick, T., Huang, X.F., 2004. Selective antagonist [H-3]SR141716A binding to cannabinoid CB1 receptors is increased in the anterior cingulate cortex in schizophrenia. Progress in Neuro-Psychopharmacology \& Biological Psychiatry 28, 355-360.

Zavitsanou, K., Huang, X.F., 2002. Decreased [H-3]spiperone binding in the anterior cingulate cortex of schizophrenia patients: An autoradiographic study. Neuroscience 109, 709-716.

Zavitsanou, K., Ward, P.B., Huang, X.F., 2002. Selective alterations in ionotropic glutamate receptors in the anterior cingulate cortex in schizophrenia. Neuropsychopharmacology 27, 826-833. 


\section{Figure Legend}

Figure 1: Means \pm one standard deviation (vertical line) of cingulum volumes for cannabis users and non-users in the two diagnostic groups (ARMS/FEP) 
Table 1: Socio-demographic characteristics ${ }^{\S}$ of ARMS and FEP

\begin{tabular}{llllll}
\hline & \multicolumn{2}{c}{$\operatorname{ARMS}(n=37)$} & \multicolumn{2}{c}{$\operatorname{FEP}(n=23)$} & Significance value \\
\hline Men & $n=22$ & $(36.7 \%)$ & $n=17$ & $(28.3 \%)$ & $\chi^{2}(1)=1.3 ; p=0.25$ \\
Women & $n=15$ & $(25 \%)$ & $n=6$ & $(10 \%)$ & \\
$\begin{array}{r}\text { Cannabis Use } \\
\text { No use }\end{array}$ & $n=22$ & $(36.7 \%)$ & $n=15$ & $(25 \%)$ & $\chi^{2}(4)=0.48 ; p=0.98$ \\
$\begin{array}{r}\text { More seldom } \\
\text { than monthly }\end{array}$ & $n=3$ & $(5 \%)$ & $n=1$ & $(1.7 \%)$ & \\
$\begin{array}{r}\text { More than } \\
\text { monthly }\end{array}$ & $n=2$ & $(3.3 \%)$ & $n=1$ & $(1.7 \%)$ & \\
$\begin{array}{r}\text { More than } \\
\text { weekly }\end{array}$ & $n=4$ & $(6.7 \%)$ & $n=2$ & $(3.3 \%)$ \\
Daily & $n=6$ & $(10 \%)$ & $n=4$ & $(6.7 \%)$
\end{tabular}

Alcohol Use

\begin{tabular}{|c|c|c|c|c|c|}
\hline No use & $n=19$ & $(31.7 \%)$ & $n=12$ & $(20 \%)$ & $\chi^{2}(4)=7.20 ; p=0.13$ \\
\hline $\begin{array}{l}\text { More seldom } \\
\text { than monthly }\end{array}$ & $n=9$ & $(15 \%)$ & $n=4$ & (6.7) & \\
\hline $\begin{array}{l}\text { More than } \\
\text { monthly }\end{array}$ & $n=3$ & $(5 \%)$ & $n=3$ & $(5 \%)$ & \\
\hline $\begin{array}{r}\text { More than } \\
\text { weekly }\end{array}$ & $n=6$ & $(10 \%)$ & $n=1$ & $(1.7 \%)$ & \\
\hline Daily & $n=0$ & $(0 \%)$ & $n=3$ & $(5 \%)$ & \\
\hline $\begin{array}{l}\text { ntipsychotic } \\
\text { nedication }\end{array}$ & $n=4$ & $(10.8 \%)$ & $n=9$ & $(39.1 \%)$ & $\chi^{2}(2)=14.2 ; p=0.001$ \\
\hline Age & 25.15 & (6.32) & 27.27 & $(6.47)$ & $t(58)=-1.25 ; p=0.22$ \\
\hline $\begin{array}{l}\text { Education (in } \\
\text { years) }\end{array}$ & 10.81 & (2.79) & 10.0 & (3.22) & $t(58)=1.03 ; p=0.30$ \\
\hline BPRS total & 39.35 & $(8.89)$ & 52.52 & $(14.36)$ & $t(56)=-4.33 ; p=0.00$ \\
\hline SANS total & 27.0 & $(17.87)$ & 35.7 & $(20.49)$ & $t(57)=-1.71 ; p=0.09$ \\
\hline $\begin{array}{l}\text { Whole brain } \\
\text { volume [means } \\
\text { in } \mathrm{mm}^{3} \text { ] }\end{array}$ & 1286748 & (113674) & 1307844 & (115520) & $t(58)=-.69 ; p=0.49$ \\
\hline \multirow{2}{*}{$\begin{array}{l}\text { Anterior } \\
\text { cingulum left }\end{array}$} & \multicolumn{2}{|c|}{$4822(1274)$} & \multicolumn{2}{|l|}{5095 (1743) } & $t(58)=-0.70 ; p=0.45$ \\
\hline & \multicolumn{2}{|c|}{$\begin{array}{l}\text { Cannabis users: } 4263 \text { (1092) } \\
\text { Non-users: } 5204 \text { (1269) }\end{array}$} & \multicolumn{2}{|c|}{$\begin{array}{l}\text { Cannabis users: } 4730 \text { (1705) } \\
\text { Non-users: } 5290 \text { (1788) }\end{array}$} & \\
\hline \multirow{3}{*}{$\begin{array}{l}\text { Anterior } \\
\text { cingulum right }\end{array}$} & \multicolumn{2}{|c|}{$4976(1647)$} & \multicolumn{2}{|l|}{$4668(1054)$} & $t(58)=0.80 ; p=0.43$ \\
\hline & \multicolumn{2}{|c|}{ Cannabis users: 5252 (1720) } & \multicolumn{2}{|c|}{ Cannabis users: 4688 (1301) } & \\
\hline & Non-users: 478 & 39 (1607) & \multicolumn{2}{|c|}{ Non-users: 4657 (947) } & \\
\hline \multirow{2}{*}{$\begin{array}{l}\text { Posterior } \\
\text { cingulum left }\end{array}$} & \multicolumn{2}{|l|}{4315 (642) } & \multirow{2}{*}{\multicolumn{2}{|c|}{$\begin{array}{l}4256(894) \\
\text { Cannabis users: }\end{array}$}} & $t(58)=0.29 ; p=0.77$ \\
\hline & \multicolumn{2}{|c|}{ Cannabis users: 41} & & & \\
\hline
\end{tabular}


Posterior

4327 (939)

cingulum right

Cannabis users: $4137(1159)$

$4425(745)$

$t(58)=-0.43 ; p=0.67$

Cannabis users: 4185 (663)

Non-users: 4554 (775)

${ }^{\S}$ Unless indicated otherwise values are given as means with SD in parentheses. 
Table 2: Repeated measures ANCOVA integrating hemisphere (left/right) and region (anterior/posterior cingulum) as within subjects variables and group (ARMS/FEP) and cannabis use (yes/no) as between subjects variables. Age, sex, medication, whole brain volume and alcohol use as covariates.

\begin{tabular}{|c|c|c|c|c|c|c|}
\hline & \multicolumn{3}{|c|}{ Anterior Cingulum } & \multicolumn{3}{|c|}{ Posterior Cingulum } \\
\hline & Partial $\eta^{2}$ & $F(d f)$ & $p$ & Partial $\eta^{2}$ & $F(d f)$ & $p$ \\
\hline Age & 0.00 & $0.08(1,51)$ & 0.77 & 0.10 & $5.61(1,51)$ & $0.02^{*}$ \\
\hline Sex & 0.00 & $0.01(1,51)$ & 0.91 & 0.07 & $3.62(1,51)$ & 0.06 \\
\hline Whole brain volume & 0.00 & $0.01(1,51)$ & 0.91 & 0.04 & $1.94(1,51)$ & 0.17 \\
\hline Alcohol use & 0.00 & $0.14(1,51)$ & 0.71 & 0.05 & $2.50(1,51)$ & 0.12 \\
\hline Medication & 0.01 & $0.37(1,51)$ & 0.55 & 0.03 & $1.56(1,51)$ & 0.22 \\
\hline Group & 0.00 & $0.13(1,51)$ & 0.72 & 0.01 & $0.67(1,51)$ & 0.41 \\
\hline Cannabis use & 0.02 & $0.80(1,51)$ & 0.37 & 0.15 & $8.96(1,51)$ & $0.00^{*}$ \\
\hline Hemisphere & 0.00 & $0.02(1,51)$ & 0.90 & 0.13 & $7.49(1,51)$ & $0.01^{*}$ \\
\hline Group * cannabis use & 0.00 & $0.03(1,51)$ & 0.85 & 0.01 & $0.43(1,51)$ & 0.52 \\
\hline Hemisphere * age & 0.02 & $0.85(1,51)$ & 0.36 & 0.01 & $0.32(1,51)$ & 0.58 \\
\hline Hemisphere * sex & 0.01 & $0.62(1,51)$ & 0.43 & 0.01 & $0.60(1,51)$ & 0.44 \\
\hline Hemisphere * whole brain & 0.01 & $0.40(1,51)$ & 0.53 & 0.11 & $6.55(1,51)$ & $0.01^{*}$ \\
\hline Hemisphere * alcohol use & 0.01 & $0.56(1,51)$ & 0.45 & 0.04 & $2.21(1,51)$ & 0.14 \\
\hline Hemisphere * medication & 0.01 & $0.25(1,51)$ & 0.62 & 0.01 & $0.14(1,51)$ & 0.71 \\
\hline Hemisphere * group & 0.01 & $0.31(1,51)$ & 0.58 & 0.01 & $0.28(1,51)$ & 0.60 \\
\hline Hemisphere * cannabis use & 0.07 & $3.50(1,51)$ & 0.05 & 0.00 & $0.00(1,51)$ & 0.97 \\
\hline Hemisphere * group * & 0.01 & $0.27(1,51)$ & 0.60 & 0.00 & $0.03(1,51)$ & 0.86 \\
\hline cannabis use & & & & & & \\
\hline
\end{tabular}




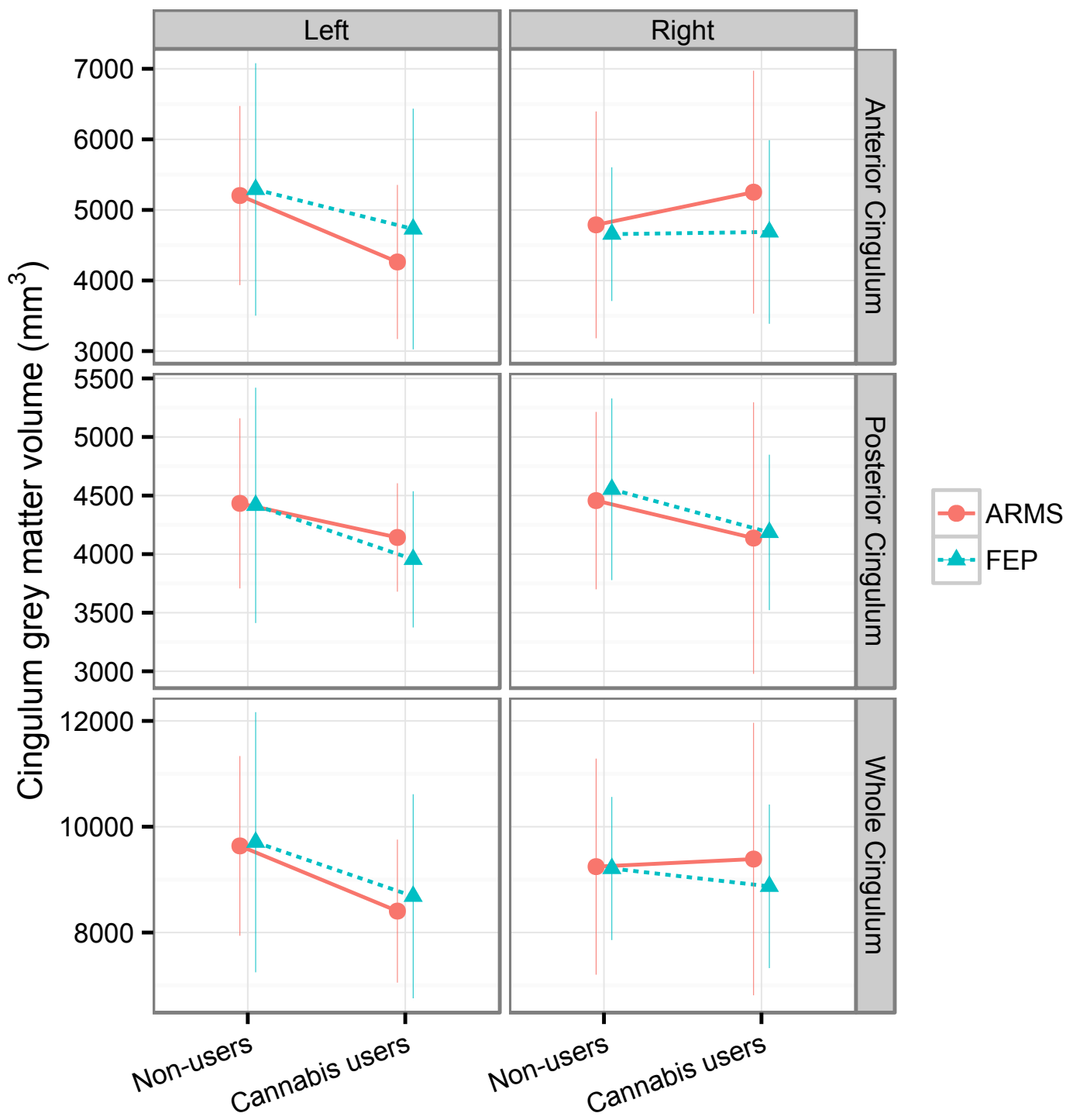




\section{Online supplementary document 1: Cingulum tracing protocol (according to}

Röthlisberger et al. (2012))

\section{Cannabis use and brain structural alterations of cingulate cortex in early psychosis}

\section{Charlotte Rapp, Anna Walter, Hilal Bugra, Erich Studerus, Corinne Tamagni, Michel Röthlisberger, Stefan Borgwardt, Jacqueline Aston, Anita Riecher- Rössler*}

This material supplements but does not replace the content of the peer-reviewed paper published in Psychiatry Research: Neuroimaging.

We focused upon anterior (ACC) and posterior cingulate cortex (PCC) both right and left as our Region-of-interest (ROI). ACC underwent a further segmentation in functional subdivisions, particularly rostral anterior (RACC), caudal anterior (CACC) and subgenual cingulate cortex (SCC), both right and left. Cingulate volume was calculated by summing up all the marked voxels, using the software for medical imaging amira ${ }^{\mathrm{TM}}$, which displays all three planes simultaneously. To reach an equal tracing of those ROl's, for each and every patient the same approach was used including clearly defined landmarks and lines. Tracing was made individually with a mouse-driven cursor for each sagittal and coronal plane. Preceding anatomical definition of the ACC and PCC was made by using Talairach CoPlanar Stereotaxic Atlas (Talairach et al., 1993). Before tracing began, several reference lines needed to be drawn on the most medial sagittal slice of each hemisphere. Two landmarks where used to reach a standard segmentation (Figure 1):

CA-CP-Line: this line passes through the superior edge of the anterior commissure and the inferior edge of the posterior commissure. It follows a path essentially parallel to the hypothalamic sulcus, dividing the thalamic from the sub-thalamic region. This line defines the horizontal plane (Talairach et al., 1993).

VCA-Line: this line is a vertical transversing the posterior margin of the anterior commissure.

This line is the basis for the vertical plane (Talairach et al., 1993). 


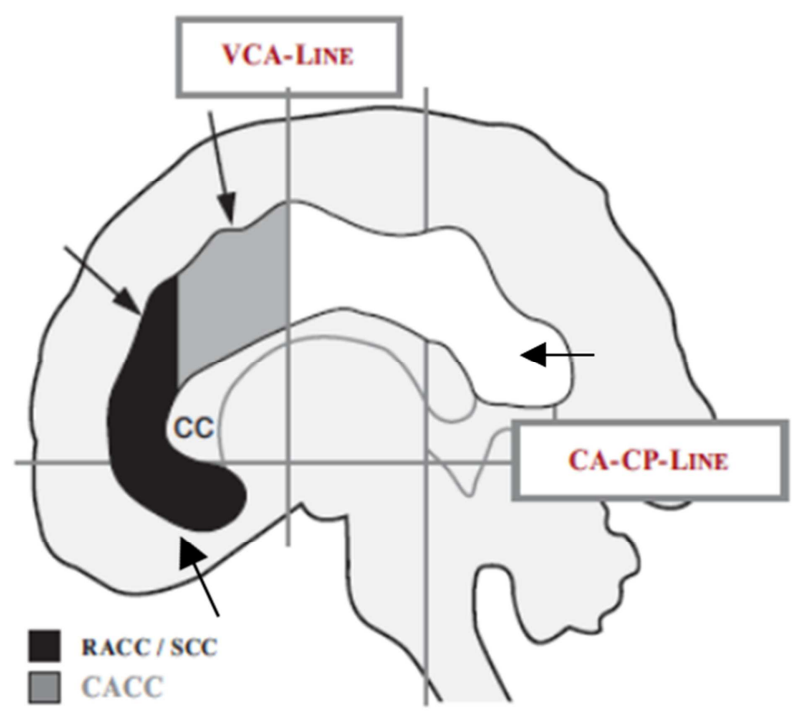

Figure 1

By setting the VCA-line with the grid-function offered by the medical imaging software amira $^{\mathrm{TM}}$ at the most superior-posterior edge of the Commissura anterior, we were able to reach a reference-associated segmentation of the cingulate gyrus in an anterior and a posterior part (Figure 1).

For a standard definition of the subdivision of the ACC, a line, defined by the coronal plane passing through the most anterior tip of the inner surface of the genu of the corpus callosum, was used as the border between the RACC and the CACC on one hand. A different line, which was set by using the coronal plane passing through the most anterior tip of the outer surface of the genu of the corpus callosum, served as border between the RACC and the SCC (Figure 1 and 2). This was described before by Crespo-Facorro et al. (1999) (CrespoFacorro et al., 1999).

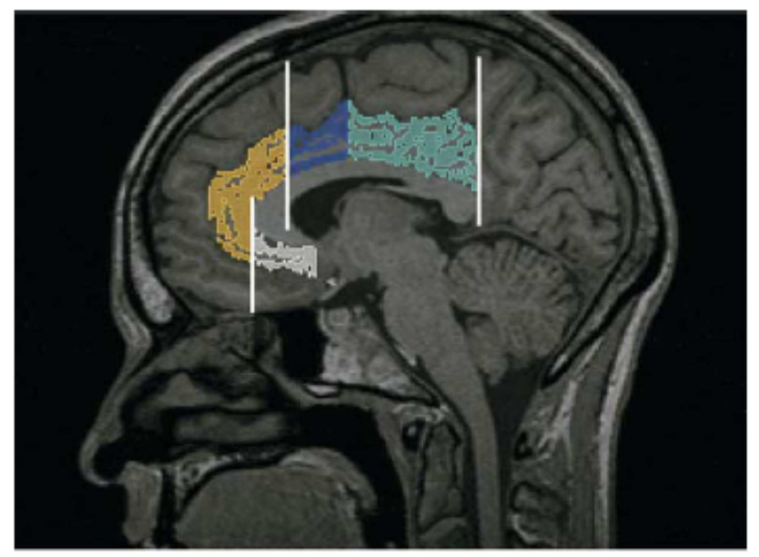

Right Cingulate Cortex: white: right SCC; yellow: right RACC, blue: right CACC; cyan: right PCC

Figure 2 
The PCC was bounded by the VCA-line on the one side (as mentioned to be the border between ACC and PCC), and by a line, which was found by the coronal plane touching the most posterior tip of the outer surface of the splenium of the corpus callosum. This line was used to have an equal reference for the dorsal border of the PCC.

Using the most sagittal plane of each hemisphere and considering the reference lines described before, the four ROl's (both left and right) were marked by with a mouse-driven cursor in eight different colours (Figure 1). The paracingulate gyrus was considered to be a part of the anterior cingulate gyrus, as assumed commonly and practiced by several studies before (Crespo-Facorro et al., 1999; Koo et al., 2008). For an accurate assessment and tracing of the gray matter associated to the overall eight different ROl's, tracing was made in a serial of coronal planes starting on the first plane showing marked RACC tissue (defined in the sagittal plane before) and was continued caudally. With this strategy, the area defined in the sagittal plane was consequently extended in each coronal plane to gain a threedimensional information of the cingulate gray matter volume. The deepest point of the callosal sulcus and the most medial point of the dorsal bank of the cingulate sulcus were used as the inner and the outer boundaries of the ACC and the PCC in each coronal slice. If there was a paracingulate gyrus, the next superior sulcus associated to its gray matter was used as upper border (Figure 2).

Crespo-Facorro, B., Kim, J., Andreasen, N., O'Leary, D., Wiser, A., Bailey, J., Harris, G., Magnotta, V., 1999. Human frontal cortex: an MRI-based parcellation method. Neuroimage 10, 500-519. Koo, M., Levitt, J., Salisbury, D., Nakamura, M., Shenton, M., McCarley, R., 2008. A cross-sectional and longitudinal magnetic resonance imaging study of cingulate gyrus gray matter volume abnormalities in first-episode schizophrenia and first-episode affective psychosis. Arch Gen Psychiatry 65, 746-760.

Röthlisberger, M., Riecher-Rossler, A., Aston, J., Fusar-Poli, P., Radu, E.-W., Borgwardt, S., 2012. Cingulate Volume Abnormalities in Emerging Psychosis. Current Pharmaceutical Design 18, 495-504. Talairach, J., Missir, O., Tournoux, P., 1993. Referentially oriented cerebral MRI anatomy: an atlas of stereotaxic anatomical correlations for gray and white matter. Thieme Medical Publishers, New York. 